Supplement of Clim. Past, 16, 2325-2341, 2020 https://doi.org/10.5194/cp-16-2325-2020-supplement

(C) Author(s) 2020. This work is distributed under the Creative Commons Attribution 4.0 License.

(c) (1)

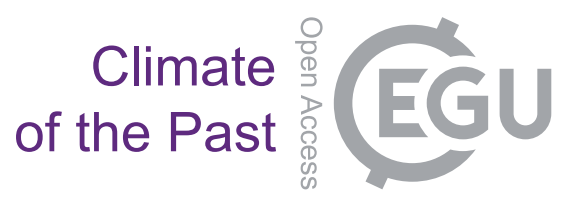

Supplement of

\title{
Evaluation of Arctic warming in mid-Pliocene climate simulations
}

Wesley de Nooijer et al.

Correspondence to: Qiong Zhang (qiong.zhang@ natgeo.su.se)

The copyright of individual parts of the supplement might differ from the CC BY 4.0 License. 
Table S1: The reconstructions used in this work for the data-model comparison of mean annual surface air temperature anomalies.

\begin{tabular}{|c|c|c|c|c|c|c|c|c|c|}
\hline $\begin{array}{l}\text { Lat } \\
\left({ }^{\circ} \mathbf{N}\right)\end{array}$ & $\begin{array}{l}\text { Lon } \\
\left({ }^{\circ} \mathbf{E}\right)\end{array}$ & $\begin{array}{l}\text { MAT } \\
\left({ }^{\circ} \mathbf{C}\right)\end{array}$ & $\begin{array}{l}\text { Observ } \\
\text { ation } \\
\left({ }^{\circ} \mathrm{C}\right)\end{array}$ & $\begin{array}{l}\text { Age } \\
\text { min } \\
\text { (Ma) }\end{array}$ & $\begin{array}{l}\text { Age max } \\
\text { (Ma) }\end{array}$ & Methods & $\begin{array}{l}\text { Uncerta } \\
\text { inty }\end{array}$ & $\begin{array}{l}\text { Confi } \\
\text { dence }\end{array}$ & Reference \\
\hline 80 & -85 & -7.1 & -14.1 & 3 & 4 & $\begin{array}{l}\text { Fossil } \\
\text { Insects } \\
\text { Assemblage } \\
\text { s }\end{array}$ & $>5$ & 4 & $\begin{array}{l}\text { Elias and Matthews, } \\
\text { 2002; Elias and } \\
\text { Kuzmina, 2013. } \\
\text { Estimates from } \\
\text { Ballantyne et al., } \\
\text { 2013. }\end{array}$ \\
\hline 79.85 & -99.24 & 4.3 & -2.9 & 2.6 & 3.6 & CRACLE & $>1.1$ & 2 & Fletcher et al., 2017 \\
\hline 78.55 & -82.33 & 1.2 & -17.2 & 3 & 4 & CRACLE & $>1.1$ & 1 & $\begin{array}{l}\text { Fletcher et al., } 2017 . \\
\text { Age from } \\
\text { Rybczynski et al. } \\
2013 .\end{array}$ \\
\hline 78.3 & -80.2 & -0.5 & -21.0 & 3 & 4 & $\begin{array}{l}\text { Tree ring } \\
\text { d18O, CA, } \\
\text { Bacterial } \\
\text { tetraether } \\
\text { composition } \\
\text { in paleosols }\end{array}$ & 1.9 & 1 & $\begin{array}{l}\text { Ballantyne et al., } \\
2010 .\end{array}$ \\
\hline 78.3 & -79.2 & 4 & -19.7 & 3.1 & 4.8 & CRACLE & $>1.1$ & 3 & $\begin{array}{l}\text { Fletcher et al., } 2017 . \\
\text { Age from } \\
\text { Rybczynski et al. } \\
2013 \text {. }\end{array}$ \\
\hline 72.2 & 125.97 & 1.5 & -15.3 & $\begin{array}{l}\text { Late } \\
\text { Pliocene }\end{array}$ & $\begin{array}{l}\text { Late } \\
\text { Pliocene }\end{array}$ & QualEst & 1 & 4 & $\begin{array}{l}\text { Fradkina 1991, } \\
\text { estimates from } \\
\text { Salzmann et al., } \\
2013 .\end{array}$ \\
\hline 69.5 & 161.5 & 2 & -8.8 & $\begin{array}{l}\text { Pliocene } \\
\text {, prior to } \\
2.4-2.5 \\
\mathrm{Ma}\end{array}$ & $\begin{array}{l}\text { Pliocene, } \\
\text { prior to } \\
2.4-2.5 \\
\mathrm{Ma}\end{array}$ & $\mathrm{CA}^{\wedge}$ & 4 & 4 & Fradkina 1991 \\
\hline 66.5 & 14.3 & 9.5 & 0.3 & 3.14 & 3.6 & $\begin{array}{l}\text { Pollen } \\
\text { Records, } \\
\text { CA }\end{array}$ & 5 & 3 & Panitz et al., 2016. \\
\hline 65.5 & -144.08 & 3 & -6.3 & 3 & 3.6 & QualEst & NA & 3 & $\begin{array}{l}\text { Ager, 1994; Ager et } \\
\text { al., 1994, estimates } \\
\text { from Salzmann et } \\
\text { al., } 2013 \text {. }\end{array}$ \\
\hline 64.53 & -149.08 & 3 & -3.8 & 2.8 & 3.6 & QualEst & 2 & 3 & $\begin{array}{l}\text { Ager, 1994; Ager et } \\
\text { al., 1994, estimates } \\
\text { from Salzmann et } \\
\text { al., } 2013 \text {. }\end{array}$ \\
\hline 64.06 & -141.95 & 7.4 & -7.7 & 2.5 & 3.3 & QualEst & NA & 3 & $\begin{array}{l}\text { Ager, 1994; Ager et } \\
\text { al., 1994, estimates } \\
\text { from Salzmann et } \\
\text { al., } 2013 \text {. }\end{array}$ \\
\hline 64 & 162 & 5.3 & -13.4 & $\begin{array}{l}\text { Late } \\
\text { Pliocene } \\
\end{array}$ & $\begin{array}{l}\text { Late } \\
\text { Pliocene } \\
\end{array}$ & $\mathrm{CA}^{\wedge}$ & $>4$ & 3 & Popova et al., 2012. \\
\hline 64 & -139 & 6.4 & -8.5 & 2.6 & 3.3 & $\mathrm{CA}^{\wedge}$ & 5 & 3 & $\begin{array}{l}\text { Pound et al., } 2015 . \\
\text { Age from Westgate } \\
\text { et al., } 2002 .\end{array}$ \\
\hline 63 & 133 & 7.4 & -13.0 & $\begin{array}{l}\text { Late } \\
\text { Pliocene }\end{array}$ & $\begin{array}{l}\text { Late } \\
\text { Pliocene }\end{array}$ & $\mathrm{CA}^{\wedge}$ & $>4$ & 3 & Popova et al., 2012. \\
\hline 60 & 150.65 & 2 & -5.9 & $\begin{array}{l}\text { Late } \\
\text { Pliocene }\end{array}$ & $\begin{array}{l}\text { Late } \\
\text { Pliocene }\end{array}$ & QualEst & NA & 4 & $\begin{array}{l}\text { Fradkina } 1991, \\
\text { estimates from } \\
\text { Salzmann et al., } \\
2013 .\end{array}$ \\
\hline
\end{tabular}


Table S2: Simulated global and Arctic $\left(60-90^{\circ} \mathrm{N}\right)$ SAT. SST and SIE anomalies (mPWP minus pre-industrial) for each model and the MMMs. Changes in maximum AMOC strength obtained from (Zhang et al., 2020).

\begin{tabular}{|c|c|c|c|c|c|c|c|}
\hline Model & $\begin{array}{l}\text { Global } \\
\text { dSAT }\left({ }^{\circ} \mathrm{C}\right)\end{array}$ & $\begin{array}{l}\text { Arctic } \\
\text { dSAT 60- } \\
90^{\circ} \mathbf{N}\left({ }^{\circ} \mathrm{C}\right) \\
\end{array}$ & $\begin{array}{l}\text { Arctic } \\
\text { amplificati } \\
\text { on ratio }\end{array}$ & $\begin{array}{l}\text { Global } \\
\text { dSST }\left({ }^{\circ} \mathrm{C}\right) \\
\end{array}$ & $\begin{array}{l}\text { Arctic dSST } \\
60-90^{\circ} \mathrm{N}\left({ }^{\circ} \mathrm{C}\right) \\
\end{array}$ & $\begin{array}{l}\text { Arctic sea ice } \\
\text { extent anomaly } \\
\left(1^{6} \mathbf{k m}^{2}\right)\end{array}$ & $\begin{array}{l}\text { Change in } \\
\text { maximum } \\
\text { AMOC } \\
\text { strength }\end{array}$ \\
\hline CCSM4-NCAR & 2.7 & 6.8 & 2.6 & 1.7 & 1.6 & -4.5 & 0.3 \\
\hline CCSM4-Utrecht & 4.8 & 10.5 & 2.2 & 3.4 & 3.0 & -7.2 & 2.1 \\
\hline CCSM4-UoT & 3.8 & 9.9 & 2.6 & 2.7 & 3.0 & -10.4 & 0.9 \\
\hline CESM1.2 & 4.1 & 9.6 & 2.4 & 2.8 & 3.1 & -5.9 & 0.3 \\
\hline CESM2 & 5.2 & 10.8 & 2.1 & 3.9 & 3.7 & -6.2 & 4.8 \\
\hline COSMOS & 3.4 & 7.2 & 2.1 & 2.1 & 2.2 & -4.4 & 3.4 \\
\hline EC-Earth 3.3 & 4.9 & 11.6 & 2.4 & 3.4 & 4.6 & -9.8 & 3.2 \\
\hline GISS-E2-1-G & 2.1 & 3.7 & 1.8 & 1.5 & 1.3 & -5.3 & 6.9 \\
\hline HadCM3 & 2.9 & 5.1 & 1.8 & 1.8 & 1.7 & -4.8 & 5.3 \\
\hline IPSLCM5A & 2.3 & 5.1 & 2.2 & 1.6 & 1.4 & -3.4 & 4.6 \\
\hline IPSLCM5A-2.1 & 2.2 & 4.8 & 2.2 & 1.6 & 1.4 & -3.1 & 5.9 \\
\hline IPSL-CM6A-LR & 3.2 & 7.0 & 2.2 & 2.4 & 2.8 & -5.2 & 3.1 \\
\hline MIROC4m & 3.1 & 7.1 & 2.3 & 2.1 & 2.2 & -5.9 & 0.6 \\
\hline MRI-CGCM2.3 & 2.4 & 7.4 & 3.1 & 1.3 & 1.4 & -4.8 & NA \\
\hline NorESM-L & 2.1 & 4.9 & 2.3 & 1.6 & 1.3 & -3.0 & 2.0 \\
\hline NorESM1-F & 1.7 & 5.2 & 3.0 & 1.3 & 1.6 & -3.4 & 3.6 \\
\hline PlioMIP2 MMM & 3.2 & 7.3 & 2.3 & 2.2 & 2.3 & -5.5 & 3.1 \\
\hline PlioMIP1 MMM & 2.7 & 6.4 & 2.4 & 1.7 & 1.5 & -5.3 & 1.5 \\
\hline
\end{tabular}

Table S3: Mean absolute deviations (MAD) and median biases for SAT $\left({ }^{\circ} \mathrm{C}\right)$ in the Arctic.

\begin{tabular}{|l|r|r|}
\hline Model & MAD $\left({ }^{\circ} \mathbf{C}\right)$ & Median bias $\left({ }^{\circ} \mathbf{C}\right)$ \\
\hline CCSM4-NCAR & 7.3 & -7.4 \\
\hline CCSM4-Utrecht & 5.6 & -4 \\
\hline CCSM4-UoT & 5.5 & -5.3 \\
\hline CESM1.2 & 6.5 & -5.0 \\
\hline CESM2 & 5.0 & -2 \\
\hline COSMOS & 6.8 & -8.1 \\
\hline EC-Earth 3.3 & 5.7 & -4.9 \\
\hline GISS-E2-1-G & 11.2 & -13.1 \\
\hline
\end{tabular}




\begin{tabular}{|l|r|r|}
\hline HadCM3 & 8.3 & -10.3 \\
\hline IPSLCM5A & 9.4 & -11.3 \\
\hline IPSLCM5A-2.1 & 9.8 & -11.8 \\
\hline IPSL-CM6A-LR & 7.8 & -9.3 \\
\hline MIROC4m & 7.4 & -9.2 \\
\hline MRI-CGCM2.3 & 8 & -9.7 \\
\hline NorESM-L & 8.5 & -6.9 \\
\hline NorESM1-F & 8.1 & -6.9 \\
\hline PlioMIP2 MMM & 7.3 & -8.2 \\
\hline PlioMIP1 MMM & 7.8 & -8.7 \\
\hline
\end{tabular}

Table S4: Global and Arctic temperature anomalies for different scenarios of future climate change simulated by CMIP5 models (Masson-Delmotte et al., 2013).

\begin{tabular}{|l|r|r|}
\hline Model & Global dSAT $\left({ }^{\circ} \mathbf{C}\right)$ & $\operatorname{Arctic}\left(\mathbf{6 7 . 5}^{-90}{ }^{\circ} \mathbf{N}\right) \operatorname{dSAT}\left({ }^{\circ} \mathbf{C}\right)$ \\
\hline RCP2.6 & 1.0 & 2.2 \\
\hline RCP4.5 & 1.8 & 4.2 \\
\hline RCP6.0 & 2.2 & 5.2 \\
\hline RCP8.5 & 3.7 & 8.3 \\
\hline
\end{tabular}

\section{References}

Ager, T.: Terrestrial palynological and paleobotanical records of Pliocene age from Alaska and Yukon Territory, Pliocene terrestrial environments and data/model comparisons: US Geological Survey Open-File Report, 94, 2-3, 1994.

Ager, T. A., Matthews Jr, J. V., and Yeend, W.: Pliocene terrace gravels of the ancestral Yukon River near Circle, Alaska: Palynology, paleobotany, paleoenvironmental reconstruction and regional correlation, Quaternary International, 22, 185-206, 1994.

Ballantyne, A., Greenwood, D., Sinninghe Damsté, J., Csank, A., Eberle, J., and Rybczynski, N.: Significantly warmer Arctic surface temperatures during the Pliocene indicated by multiple independent proxies, Geology, 38, 603-606, 2010.

Ballantyne, A. P., Axford, Y., Miller, G. H., Otto-Bliesner, B. L., Rosenbloom, N., and White, J. W.: The amplification of Arctic terrestrial surface temperatures by reduced sea-ice extent during the Pliocene, Palaeogeography, Palaeoclimatology, Palaeoecology, 386, 59-67, 2013.

Elias, S. A., and Jr., J. V. M.: Arctic North American seasonal temperatures from the latest Miocene to the Early Pleistocene, based on mutual climatic range analysis of fossil beetle assemblages, Canadian Journal of Earth Sciences, 39, 911-920, 10.1139/e01-096, 2002.

Elias, S. A., and Kuzmina, S.: BEETLE RECORDS | Late Tertiary and Early Quaternary Records, in: Encyclopedia of Quaternary Science (Second Edition), edited by: Elias, S. A., and Mock, C. J., Elsevier, Amsterdam, 173-183, 2013.

Fletcher, T., Feng, R., Telka, A. M., Matthews, J. V., and Ballantyne, A.: Floral Dissimilarity and the Influence of Climate in the Pliocene High Arctic: Biotic and Abiotic Influences on Five Sites on the Canadian Arctic Archipelago, Frontiers in Ecology and Evolution, 5, 10.3389/fevo.2017.00019, 2017. 
Fradkina, A.: Pliocene climatic fluctuations in the Far North-East of the USSR, Pliocene Climates of the Northern Hemisphere, Abstr. Joint US/USSR Workshop on Pliocene Paleoclimates, Moscow, 1990,

Masson-Delmotte, V., Schulz, M., Abe-Ouchi, A., Beer, J., Ganopolski, A., Rouco, J. G., Jansen, E., Lambeck, K., Luterbacher, J., and Naish, T.: Information from Paleoclimate Archives In: Climate Change 2013: The Physical Science Basis Contribution of Working Group I to the Fifth Assessment Report of the Intergovernmental Panel on Climate Change ed TF Stocker et al, Cambridge, United Kingdom and New York, NY, USA, 2013.

Panitz, S., Salzmann, U., Risebrobakken, B., De Schepper, S., and Pound, M. J.: Climate variability and longterm expansion of peatlands in Arctic Norway during the late Pliocene (ODP Site 642, Norwegian Sea), Clim. Past, 12, 1043-1060, 10.5194/cp-12-1043-2016, 2016.

Popova, S., Utescher, T., GROMYKO, D., BRUCH, A., and Mosbrugger, V.: Palaeoclimate evolution in Siberia and the Russian Far East from the Oligocene to Pliocene-evidence from fruit and seed floras, Turkish Journal of Earth Sciences, 21, 315-334, 2012.

Pound, M. J., Lowther, R. I., Peakall, J., Chapman, R. J., and Salzmann, U.: Palynological evidence for a warmer boreal climate in the Late Pliocene of the Yukon Territory, Canada, Palynology, 39, 91-102, 2015.

Rybczynski, N., Gosse, J. C., Richard Harington, C., Wogelius, R. A., Hidy, A. J., and Buckley, M.: MidPliocene warm-period deposits in the High Arctic yield insight into camel evolution, Nature Communications, 4, 1550, 10.1038/ncomms2516, 2013.

Salzmann, U., Dolan, A. M., Haywood, A. M., Chan, W.-L., Voss, J., Hill, D. J., Abe-Ouchi, A., Otto-Bliesner, B., Bragg, F. J., Chandler, M. A., Contoux, C., Dowsett, H. J., Jost, A., Kamae, Y., Lohmann, G., Lunt, D. J., Pickering, S. J., Pound, M. J., Ramstein, G., Rosenbloom, N. A., Sohl, L., Stepanek, C., Ueda, H., and Zhang, Z.: Challenges in quantifying Pliocene terrestrial warming revealed by data-model discord, Nature Climate Change, 3, 969-974, 10.1038/nclimate2008, 2013.

Westgate, J., Sandhu, A., Preece, S., and Froese, D.: Age of the gold-bearing White Channel gravel, Klondike district, Yukon, Yukon exploration and geology, 2002, 241-250, 2002.

Zhang, Z., Li, X., Guo, C., Otterå, O. H., Nisancioglu, K. H., Tan, N., Contoux, C., Ramstein, G., Feng, R., Otto-Bliesner, B. L., Brady, E., Chandan, D., Peltier, W. R., Baatsen, M. L. J., von der Heydt, A. S., Weiffenbach, J. E., Stepanek, C., Lohmann, G., Zhang, Q., Li, Q., Chandler, M. A., Sohl, L. E., Haywood, A. M., Hunter, S. J., Tindall, J. C., Williams, C., Lunt, D. J., Chan, W.-L., and Abe-Ouchi, A.: Mid-Pliocene Atlantic Meridional Overturning Circulation simulated in PlioMIP2, Clim. Past Discuss., https://doi.org/10.5194/cp-2020-120, 2020. 